\title{
Fachtagung „Evaluation bei der Polizei NRW“ am 23. und 24. Mai 2019 im Landeskriminalamt Nordrhein-Westfalen (LKA NRW)
}

\author{
Ein Rückblick auf 15 Jahre Evaluation in der Polizei - \\ Was gelingt gut? Was bereitet Schwierigkeiten? \\ Welche Ziele lassen sich daraus ableiten?
}

\section{Karin Sassen ${ }^{1}$}

\section{Einführung}

Anlässlich des 15. ,Geburtstages' der Zentralstelle Evaluation (ZEVA) im LKA NRW lud Behördenleiter Frank Hoever zur Fachtagung ,Evaluation bei der Polizei NRW“ ein. Rund 40 Gäste folgten der Einladung. Sie setzten sich zu etwa zwei Dritteln aus Polizeiangehörigen zusammen und zu etwa einem Drittel aus externen Fachleuten. Die Polizeibeamtinnen und Polizeibeamten kamen sowohl aus NRW als auch aus anderen Bundesländern, außerdem vom Bundeskriminalamt und der Bundespolizei. Als externe Organisationen waren neben der DeGEval beispielsweise das Nationale Zentrum für Kriminalprävention (NZK), das Kriminologische Forschungsinstitut Niedersachsen e.V. (KFN) und das Institut für Gesetzesfolgenabschätzung und Evaluation (InGFA) vertreten. Es sollte gemeinsam ein Rückblick auf die bisherige Evaluationstätigkeit in der Polizei NRW geworfen werden, um hieraus gemeinsam Ziele für die zukünftige Entwicklung abzuleiten und erste Schritte zu planen.

\section{Vorträge}

Nach der Begrüßung durch Herrn Hoever gab der Vorsitzende der DeGEval, Prof. Dr. Jan Ulrich Hense, den ersten Input. Er vermittelte zunächst evaluationstheoretisches Grundwissen; dass der Ursprung der Evaluation in den 1960er Jahren zu suchen sei, in der Ära der großen Sozialreformen, und die Evaluation ein Teil der Social Experiments in der darauffolgenden Zeit gewesen sei. Mit Wissen die Welt zu

1 Zentralstelle Evaluation, Landeskriminalamt Nordrhein-Westfalen, Düsseldorf 
verbessern, sei damals die Grundidee gewesen, die bis heute fortgelte. Eine ideale Abfolge liege vor, wenn Evaluationen Ergebnisse hervorbrächten, deren Nutzen zu einem Mehrwert führten. Weiter ging es um die vier Hauptfunktionen von Evaluation und einige Bedingungen für erfolgreiche Evaluationen (Standards einhalten, maßgeschneiderte Evaluationen, Einbeziehung von Stakeholdern, Einbettung der Evaluationsfunktion in die Organisation). Hense schloss mit dem Verweis auf John Gargani, der für die Zukunft der Evaluation den Trend zu vermehrter interner Evaluation sehe.

Auf Hense folgte Dieter Quermann, erster Leiter der ZEVA (bis 2012), der auf launige Art und Weise und gespickt mit vielen Anekdoten seine ersten Schritte beim Aufbau der neuen Dienststelle schilderte. Viele Hürden und Vorbehalte seien zu überwinden gewesen. Nur wenige Polizeibehörden hätten anfangs mit der ZEVA zusammenarbeiten wollen. Das Verständnis für den Sinn von Evaluation habe man immer wieder vermitteln müssen. Eine besondere Herausforderung stelle die berufsspezifische Prägung der Kolleginnen und Kollegen dar. Bewusst vereinfachend sagte er, dass die Polizei keine Probleme löse, sondern Lagen bewältige: sofortiges Handeln statt ursachenorientierter Problemlösung. Die ZEVA habe gemeinsam mit den Polizeibehörden den Raum für Analyse und Konzeptionierung erweitern und dadurch die Wirksamkeit polizeilichen Tuns fördern können. Genau hier liege das Potenzial für die Entwicklung von Evaluation polizeilicher Arbeit.

Dass Dieter Quermann ebenso wie sein Nachfolger Ingo Dungs die ZEVA zu einer funktionierenden Einheit entwickelt hätten, die im polizeilichen Evaluationsgeschäft eine wichtige Rolle einnehme, erläuterte Prof. Dr. Thomas Bliesener, Direktor des Kriminologischen Forschungsinstituts Niedersachsen e.V., auf humorvolle Art und aus eigener Erfahrung. Er habe die Mitarbeiterinnen und Mitarbeiter der ZEVA als diejenigen erlebt, die externe Wissenschaftlerinnen und Wissenschaftler in die Welt der Polizei einführten, die Prozessabläufe und vorhandene Datenbestände erklärten, die das interne Feld auf die Datenerhebung vorbereiteten und dabei in beide Richtungen bei ,Übersetzungsproblemen' helfen würden. Im Weiteren sei die ZEVA hilfreich bei der Interpretation von Befunden und würde schließlich noch zwischen Zuwendungsgeber und Zuwendungsempfänger moderieren, wenn das Kontrollbedürfnis auf der einen Seite der wissenschaftlichen Freiheit auf der anderen Seite entgegenstehe. Auf diese wichtige Vermittlungsfunktion sei auch in $\mathrm{Zu}$ kunft besonderes Augenmerk zu legen.

Für die aktuelle Situation stellte ZEVA-Leiter Ingo Dungs fest, dass die Aufgaben und Anforderungen zahlreicher und vielfältiger würden. Im Wesentlichen ließen sich vier Aufgabenbereiche unterscheiden:

1. Beratung der Kreispolizeibehörden in NRW und des Ministeriums des Innern (IM) NRW

2. Wissensmanagement ZEVA-intern und -extern in der Polizei

3. Auftragsvergaben für externe Evaluationen und deren Begleitung

4. Eigene wirkungsorientierte Evaluation

Das ZEVA-interne Wissensmanagement werde insbesondere durch Veranstaltungen der DeGEval geprägt, darüber hinaus durch In-House-Schulungen, Klausurtagungen und die Erfahrungen mit externen Evaluationen. Dieses Wissen werde NRW-weit 
an die Kreispolizeibehörden vermittelt. Dies geschehe beispielsweise durch Mitwirkung an Fortbildungen für Mitarbeiterinnen und Mitarbeiter in Führungsstäben, durch Mitwirkung an der Ausbildung des höheren Dienstes und durch Beratungsleistungen auf Anfrage. Gegenstände externer Evaluationen, die von der ZEVA begleitetet wurden, waren etwa SKALA (ein durch das LKA NRW entwickelter Predictive-Policing-Ansatz; vgl. Landeskriminalamt NRW 2018), § 15a PolG NRW (Videobeobachtung; vgl. Glaubitz et al. 2018) und Kurve kriegen (ein präventiver Ansatz zum Umgang mit jugendlichen Intensivtäterinnen und -tätern; vgl. Bartsch et al. 2013; Bliesener et al. 2015). Eigene Evaluationen bezogen sich auf das Haus des Jugendrechts in Paderborn (nicht veröffentlicht) sowie auf Maßnahmen in mehreren Handlungsfeldern der bundesweit ausgerichteten Initiative des IMNRW „Mehr Sicherheit bei Fußballspielen in Nordrhein-Westfalen" (vgl. Landeskriminalamt NRW 2011, 2012, 2013, 2016). Insgesamt basiere das ZEVA-interne Evaluationswissen inzwischen auf einem breiten Erfahrungsschatz. Nach außen erlebe man eine breite Akzeptanz. Luft nach oben sehe er jedoch im polizeiinternen Austausch, insbesondere auf Bundesebene. In der Bund-Länder-Gremienstruktur im Bereich Innere Sicherheit sei Evaluation bisher nicht verankert. Aktuelle Beziehungen in andere Länder bestünden aufgrund zufälliger und persönlich motivierter Kontaktpflege, die beispielsweise auf DeGEval-Tagungen und in DeGEval-Arbeitskreisen möglich sei. Wünschenswert sei ein formales Netzwerk mit eigener Agenda. Nach diesen internen wie externen Inputs ging es in die Workshops.

\section{Workshops zu Vergabe, Prozessevaluation und Netzwerkbildung}

In Workshop 1 wurden die sogenannte Projektantenproblematik in Vergabeverfahren beleuchtet und Erfahrungen aus Vergabeverfahren allgemein ausgetauscht. Den rechtlichen Rahmen setzt das Vergaberecht in $\S 20 \mathrm{UVgO}$ (nationale Vergabeverfahren) beziehungsweise $\S 28 \mathrm{VgV}$ (EU-Vergabeverfahren). Im Wesentlichen sind die Regelungen darauf ausgerichtet, einen fairen, diskriminierungsfreien und transparenten Wettbewerb herzustellen und Korruption zu verhindern. Aus Sicht von öffentlichen Auftraggebenden ist festzuhalten, dass eine Markterkundung zur Vorbereitung eines Vergabeverfahrens (zum Beispiel einer öffentlichen Ausschreibung) erfolgen kann und von daher im Vorfeld der Ausschreibung schon die Möglichkeit besteht, mit potenziellen Bieterinnen und Bietern Kontakt aufzunehmen. Im Verlauf des Vergabeverfahrens sei dann transparente Kommunikation wesentlicher Bestandteil einer fairen, diskriminierungsfreien und transparenten Vergabe. Bewährt hätten sich mehrstufige Verfahren. Hinsichtlich der Konkretheit von Leistungsbeschreibungen habe sich meist ein Mittelweg bewährt. Anzuwendende Methoden sollten nicht vorbestimmt werden. Das zu untersuchende Programm oder Projekt muss so konkret wie möglich beschrieben werden. Der Ausschluss einer Bieterin oder eines Bieters wegen Beratungstätigkeit im Vorfeld sei nur als ultima ratio denkbar und komme erst zum Tragen, wenn das Sonderwissen des Projektanten nicht ausgeglichen werden könne. 
Workshop 2 thematisierte die Bedeutung von Prozessevaluationen für Wirkungsevaluationen. Diskutiert wurde über die Qualität polizeilicher Konzepte, über die Bedeutung von Wirkungslogiken und Folgen nicht konzepttreuer Umsetzung im polizeilichen Alltag. Vor diesem Hintergrund war die Erreichbarkeit von Wirkungsnachweisen Gegenstand der Überlegungen. Als besondere Herausforderung stelle sich die polizeiliche Mentalität dar, Probleme immer sofort lösen zu wollen. Einer sorgfältigen Ursachenanalyse und der Entwicklung einer Zielarchitektur mit passgenauen Maßnahmen werde meist wenig Raum gegeben. Hier biete sich in der Polizei Entwicklungspotenzial. Eine besondere Aufgeschlossenheit gegenüber evaluationsspezifischen Denkansätzen in der Projektentwicklung sei aktuell bei jungen Führungskräften zu beobachten.

Ziel des Workshops 3 war es, Möglichkeiten zu erarbeiten, die die innerpolizeiliche Vernetzung zum Thema Evaluation stärken. Breite Unterstützung fand die Idee, polizeiintern den Begriff Evaluation bekannter zu machen und dabei von benachbarten Disziplinen wie beispielsweise Controlling und Qualitätssicherung abzugrenzen. Dies schaffe ein besseres Verständnis von Evaluation, welches nach Einschätzung der Teilnehmenden in der Polizei weithin noch fehle. Für eine begleitende Öffentlichkeitsarbeit könnten unter anderem öffentlich zugängliche Polizeizeitschriften genutzt werden, wie zum Beispiel die „Streife“, das Magazin des IM NRW für die nordrhein-westfälische Polizei. Auch für eine bessere Präsenz von Evaluation auf polizeiinternen Intranetseiten (auf Länder- sowie auf Bundesebene) wurden Ideen entwickelt. Schließlich fand die Überlegung Anklang, ein bundesweites Netzwerk zum Austausch von Erfahrungen zu etablieren.

\section{Podiumsdiskussion zum Umgang mit Evaluationsergebnissen im politischen Raum}

Der Einladung des LKA NRW zur Podiumsdiskussion waren Dr. Andreas Armborst (Nationales Zentrum für Kriminalprävention, NZK), Prof. Dr. Thomas Bliesener (Kriminologisches Forschungsinstitut Niedersachsen, KFN), Prof. Dr. Wolfgang Böttcher (Westfälische Wilhelms-Universität Münster), Dr. Susanne Giel (Univation), Prof. Dr. Stefan Kersting (Fachhochschule für öffentliche Verwaltung NRW, ab Januar 2020 Hochschule für Polizei und öffentliche Verwaltung NRW), Dr. Hartmut Pfeiffer (LKA Niedersachsen) und Dr. Stefan Silvestrini (CEval) gefolgt. Als Ausgangspunkt diente die Frage nach Chancen und Risiken im Umgang mit den Ergebnissen einer Evaluation im politischen Raum.

Einleitend wurde zunächst betont, dass bei politischen Entscheidungsträgerinnen und Entscheidungsträgern ein zunehmendes Bewusstsein für die Bedeutung von Evaluationen für den politischen Diskurs zu beobachten sei. Die Frage, ob Mittel sinnvoll investiert worden seien und Maßnahmen tatsächlich Wirkung zeigten, werde häufiger gestellt. Diese Denkweise habe in den USA bereits eine lange Tradition, während sie hier noch im Aufkeimen sei. 
Als günstige Zeitpunkte für Evaluationen wurde auf Zeiten des Regierungswechsels oder des Generationswechsels hingewiesen. Allgemein seien Zeiten des Paradigmenwechsels opportun; Zeiten, in denen alles auf den Prüfstand gestellt werde.

Speziell in Bezug auf polizeiliche Konzepte und der Frage, warum diese zu evaluieren seien, forderte Kersting dazu auf, insbesondere die Perspektive der Bürgerinnen und Bürger einzubeziehen. Polizeiliche Maßnahmen griffen - auf Basis von Gesetzen - in Grundrechte ein. Die Polizei sei die Institution, die das Gewaltmonopol des Staates durchsetze. Damit sei sie auch diejenige Institution, die ständig in Grundrechte eingreife, auch in Form polizeilicher Konzepte. Ein solcher Eingriff sei rechtmäßig, wenn entsprechende gesetzliche Voraussetzungen vorlägen. Aus verfassungsrechtlicher Sicht seien Grundrechtseingriffe jedoch auf ein Minimum zu beschränken. Voraussetzung für die Rechtmäßigkeit eines Eingriffs sei, dass die Maßnahme, die in die Grundrechte eingreife, geeignet sei, um ein bestimmtes Ziel zu erreichen. Zugespitzt könne man den Standpunkt vertreten, dass Vorschriften mit Eingriffscharakter evaluiert werden müssten, um deren Geeignetheit nachzuweisen. Andernfalls seien verfassungsrechtliche Bedenken angebracht. Daher sollte bei der Evaluation polizeilicher Eingriffskonzepte grundsätzlich die Perspektive über Polizei und Politik hinaus auch auf den Bereich der Zivilgesellschaft erweitert werden. Diese müsste das Bedürfnis nach Evaluation von der Politik einfordern.

Des Weiteren wurde lebhaft zur Leistungsfähigkeit und Aussagekraft von Evaluationen diskutiert. Unter anderem wurde der Standpunkt vertreten, Wirkung könne gar nicht gemessen werden. Es würden vielmehr Veränderungen gemessen und Zustände verglichen. Über eine Wirkungslogik könne möglicherweise eine Veränderung einer Maßnahme zugeordnet werden. Oder man könne versuchen zu quantifizieren, welchen Beitrag eine Maßnahme zu einer Veränderung beigetragen habe. Hieran anschließend erläuterte Böttcher sein Verständnis von ,Evidence' und dass genau dieses Verständnis für die Bestimmtheit von Evaluationen elementar sei. Die Bedeutung von ,Evidence' könne man beim Anschauen der forensisch Tätigen von CSI (amerikanische Krimiserie) erfahren. Diese sammelten DNA, Fingerabdrücke und zahlreiche andere Beweismittel. Aus diesen Beweismitteln würden sodann Richterschaft und Verteidigung vor Gericht unterschiedliche Geschichten erzeugen. Auch Evaluatorinnen und Evaluatoren arbeiteten vergleichbar. Auch sie produzierten ,Evidence' (,Beweismittel'), aber keine Evidenz im Sinne einer ,Offensichtlichkeit' und Eindeutigkeit. Aus den Beweismitteln ,komponiere" man dann eine Aussage über Prozesse und Wirkungen.

Zur Frage, inwieweit ein Recht oder eine Pflicht bestehe, Evaluationen zu veröffentlichen, wurden je nach Perspektive und Organisationszugehörigkeit unterschiedliche Standpunkte vertreten. Externe Evaluatorinnen und Evaluatoren, wie beispielsweise Bliesener, fordern ihr Recht auf Veröffentlichung zum Teil ein und verzichten eher auf einen Auftrag als von diesem Recht abzurücken. Nach anderer Meinung müsse aber auch die Möglichkeit bestehen, Evaluationen mit rein interner Zielrichtung durchführen zu können und Ergebnisse allein innerhalb einer Organisation zu kommunizieren und zu nutzen.

Im Umgang mit Ergebnissen, die auf ausbleibende Wirkung von Maßnahmen hinweisen, wird ein noch ausstehender Lernprozess auf Seiten der Auftraggebenden 
beschrieben. Ein solches Evaluationsergebnis werde als schlechte Nachricht aufgefasst, obwohl es als nützlich aufgenommen werden sollte. Ein Programmabbruch könne schließlich sinnvoll sein, um Ressourcen für bessere Projekte zu schonen. Auch die Differenzierung, dass Mitarbeitende durchaus gute Arbeit geleistet haben könnten, aber das Programm schlicht nichts tauge, fiele schwer. Bliesener betonte, dass die Möglichkeit eines negativen Ergebnisses von Anfang mitgedacht und in die Planung einbezogen werden müsse. Es müsse möglich sein, aus dem Feld rauszugehen, ohne Scherben zerbrochen zu haben und ohne angefeindet zu werden. Hierfür sei ein Plan B wichtig. Auch unter diesem Aspekt befürworte er eine partizipative Evaluation, für die er viele Beteiligte mit in die Evaluation einbeziehe und mit diesen auch vor der Veröffentlichung über Ergebnisse diskutiere.

Als kleine Schritte der Verbesserung wurden genannt:

- Entwicklung eines Bewusstseins für Evaluation in der Organisation, um Momente möglich zu machen, in denen über Ziele und Wege nachgedacht wird,

- vom Nutzen her denken und den Adressatinnen und Adressaten erklären, was sie mit den Ergebnissen der Evaluation anfangen können,

- geschützte Räume für Beteiligte schaffen und Ergebnisse mit diesen im kleinen Kreis diskutieren, bevor sie veröffentlicht werden.

Eine erfolgversprechende Perspektive ergebe sich durch das EU-Programm „Secure Societies“. In diesem Zusammenhang werde stark auf Innovationen und die Förderung von Innovationen gesetzt. Die Sicherheitspolitik spiele eine wesentliche Rolle. Auf europäischer Ebene sei unstreitig, dass dort, wo Innovationen zum Tragen kommen, auch Evaluationen betrieben würden. Dies biete insgesamt die Chance, das Prinzip der Evaluation im Bereich der Sicherheitspolitik weiter zu verankern.

Armborst ergänzte aus Sicht des NZK, dass Evaluationen eine große Bedeutung im Politikfeld Innere Sicherheit haben könnten. Dafür müssten ihre Ergebnisse und Empfehlungen jedoch im politischen Diskurs anschlussfähig sein. Zum Beispiel indem sie Personen mit Entscheidungsbefugnis mit einem klaren Vokabular ausstatteten, das es ihnen erlaube, in kurzen Statements belastbare Aussagen über das evaluierte Programm zu machen. Dies sei der beste Weg, populistischen Argumenten etwas entgegenzusetzen.

\section{Fazit}

Als Veranstalterinnen und Veranstalter waren wir - die ZEVA - unglaublich dankbar für das tolle Engagement aller Teilnehmerinnen und Teilnehmer, insbesondere der Mitwirkenden der Podiumsdiskussion, für ihre hochinteressanten und ehrlichen Beiträge. Für uns haben sich viele Impulse für unsere weitere Arbeit ergeben. Wir hoffen, dass dies auch für unsere Gäste gilt und dass sich auch zukünftig Möglichkeiten zur Fortsetzung des Erfahrungsaustauschs ergeben werden. 


\section{Literatur}

Bartsch, Sina/Glaubitz, Christoffer/Hausmann, Barbara/Bliesener, Thomas (2013): Prozess- und Wirkungsevaluation der NRW-Initiative „Kurve kriegen“ - Abschlussbericht Prozessevaluation. Kiel: Christian-Albrechts-Universität. Verfügbar unter: https://kfn.de/wp-content/uploads/down loads/Abschlussbericht_Prozessevaluation_01072013.pdf [06.12.2019].

Bliesener, Thomas/Glaubitz, Christoffer/Hausmann, Barbara/Klatt, Thimna/Riesner, Lars (2015): Prozess- und Wirkungsevaluation der NRW-Initiative „Kurve kriegen“ - Abschlussbericht Wirkungsevaluation. Kiel: Christian-Albrechts-Universität. Verfügbar unter: https://www.kurvekrie gen.nrw.de/wp-content/uploads/2018/07/Abschlussbericht-Wirkungsevaluation_060315.pdf [06.12.2019].

Glaubitz, Christiopher/Kudlacek, Dominic/Neumann, Merten/Fleischer, Stephanie/Bliesener, Thomas (2018): Ergebnisse der Evaluation der polizeilichen Videobeobachtung in Nordrhein-Westfalen gemäß $§ 15 \mathrm{a}$ PolG NRW. Hannover: KFN Forschungsbericht Nr. 143. Verfügbar unter: https:// kfn.de/wp-content/uploads/Forschungsberichte/FB_143.pdf [06.12.2019].

Landeskriminalamt NRW (2011): Evaluation der polizeilichen Rahmenkonzeption zur NRW-Initiative „Mehr Sicherheit bei Fußballspielen“. Verfügbar unter: http://intrapol.polizei.nrw.de/Zentrale AufgabenFStZEVAEvaluationsberichteKPB/1.\%20Evaluationsbericht\%20NRW-Initiative\%20 Fußball.pdf [10.01.2020].

Landeskriminalamt NRW (2012): Zweite Evaluation der polizeilichen Rahmenkonzeption zur NRWInitiative „Mehr Sicherheit bei Fußballspielen“. Verfügbar unter: http://intrapol.polizei.nrw.de/ ZentraleAufgabenFStZEVAEvaluationsberichteKPB/2.\%20Evaluationsbericht $\% 20 \mathrm{NRW}$-Initiative \%20Fußball.pdf [10.01.2020].

Landeskriminalamt NRW (2013): Dritte Evaluation der polizeilichen Rahmenkonzeption zur NRWInitiative „Mehr Sicherheit bei Fußballspielen“. Verfügbar unter: http://intrapol.polizei.nrw.de/ ZentraleAufgabenFStZEVAEvaluationsberichteKPB/3.\%20Evaluationsbericht\%20NRW-Initiative \%20Fußball.pdf [10.01.2020].

Landeskriminalamt NRW (2016): Vierte Evaluation der polizeilichen Rahmenkonzeption zur NRWInitiative „Mehr Sicherheit bei Fußballspielen“ - Spürbare Reduzierung der Gastkartenkontingente bei Hochrisikospielen. Verfügbar unter: http://intrapol.polizei.nrw.de/ZentraleAufgabenFStZEVAEvaluationsberichteKPB/4.\%20Evaluationsbericht\%20NRW-Initiative\%20Fußball.pdf [10.01.2020].

Landeskriminalamt NRW (2018): Kooperative Evaluation des Projektes SKALA. Abschlussbericht der Zentralstelle Evaluation beim LKA NRW (ZEVA) und der Gesellschaft für innovative Sozialforschung und Sozialplanung e.V. Bremen (GISS). Düsseldorf. 